\title{
On linear sets on a projective line
}

\author{
M. Lavrauw G. Van de Voorde
}

\begin{abstract}
Linear sets generalise the concept of subgeometries in a projective space. They have many applications in finite geometry. In this paper we address two problems for linear sets: the equivalence problem and the intersection problem. We consider linear sets as quotient geometries and determine the exact conditions for two linear sets to be equivalent. This is then used to determine in which cases all linear sets of rank 3 of the same size on a projective line are (projectively) equivalent. In [3], the intersection problem for subgeometries of $\operatorname{PG}(n, q)$ is solved. The intersection of linear sets is much more difficult. We determine the intersection of a subline $\mathrm{PG}(1, q)$ with a linear set in $\mathrm{PG}\left(1, q^{h}\right)$ and investigate the existence of irregular sublines, contained in a linear set. We also derive an upper bound, which is sharp for odd $q$, on the size of the intersection of two different linear sets of rank 3 in $\mathrm{PG}\left(1, q^{h}\right)$.
\end{abstract}

\section{Introduction and preliminaries}

Linear sets have been intensively used in recent years in order to classify, construct or characterise various structures related to finite incidence geometry. Besides their relation to blocking sets (see below), linear sets also appear in the study of translation ovoids (see e.g. [10]), and are of high relevance in the theory of semifields (e.g. [7]).

In contrast to the number of papers that make use of linear sets, few papers have been published in which linear sets are the main object. To our knowledge the only general treatment of linear sets is by Polverino [11]. Apart from the lack of knowledge about linear sets, in particular about the possible intersections of linear sets, the main motivation for this research arose from the study of blocking sets in finite projective spaces.

If $V$ is a vectorspace, then we denote by $\mathrm{PG}(V)$ the corresponding projective space. If $V$ has dimension $n$ over the finite field $\mathbb{F}_{q}$ with $q$ elements, then we also write $\mathrm{PG}(n-1, q)$.

A small minimal $k$-blocking set is a set $B$ of points in the projective space $\mathrm{PG}(n, q)$, meeting every $n-k$-space, where $|B| \leq 3\left(q^{k}+1\right) / 2$ and such that no proper subset of $B$ is a $k$-blocking set. The term "linear" was used by Lunardon to describe a particular construction of a small minimal blocking set [9], which led to the first construction of small blocking sets that are not of Rédei type. This construction opened up a new perspective on blocking sets and soon people conjectured that all small minimal $k$-blocking sets in $\mathrm{PG}(n, q)$ must be linear. However, it took until 2008 until this so-called "Linearity conjecture" was formally stated in the literature, see Sziklai [12]. To our knowledge the Linearity conjecture is still open, apart from various instances. Recently a proof of the 
Linearity conjecture in $\mathrm{PG}\left(n, p^{3}\right), p$ prime, was given in [8], where the authors use that an $\mathbb{F}_{q}$-linear set $S$ of rank 3 and a subline of $\operatorname{PG}\left(1, q^{3}\right)$, not contained in $S$, have at most 3 points in common. This served as an extra motivation and it is our hope that the results obtained here will bring us closer to solving the Linearity conjecture for blocking sets. One of the theorems in this paper, of relevance here, determines the intersection of an $\mathbb{F}_{q}$-linear set of rank $k$ with a subline in $\mathrm{PG}\left(1, q^{h}\right)$.

We also study the possible intersections of two linear sets (Section 5). In the case where the linear sets are subgeometries this problem was recently solved in [3] by Durante and Donati (completing a study originated by Bose et al. in 1980) showing that the intersection of two subgeometries is necessarily the union of subgeometries in independent subspaces. The intersection of linear sets is considerably more difficult, and it is not necessarily the union of linear sets in independent subspaces.

Let $V$ be an $r$-dimensional vectorspace over a finite field $\mathbb{F}$. A set $S$ of points of $\mathrm{PG}(V)$ is called a linear set (of rank $t$ ) if there exists a subset $U$ of $V$ that forms a $\left(t\right.$-dimensional) $\mathbb{F}_{q}$-vectorspace for some $\mathbb{F}_{q} \subset \mathbb{F}$, such that $S=\mathcal{B}(U)$, where

$$
\mathcal{B}(U):=\left\{\langle u\rangle_{\mathbb{F}}: u \in U \backslash\{0\}\right\} .
$$

If we want to specify the subfield we call $S$ an $\mathbb{F}_{q}$-linear set. In other words, if $\mathbb{F}=\mathbb{F}_{q^{n}}$, we have the following diagram

$$
\begin{array}{ccccc}
\mathbb{F}_{q^{n}}^{r} & \longleftrightarrow & \mathbb{F}_{q}^{r n} & \supseteq & U \\
\uparrow & & \uparrow & & \uparrow \\
\mathcal{B}(U) \subseteq \operatorname{PG}\left(r-1, q^{n}\right) & \longleftrightarrow & \mathrm{PG}(r n-1, q) & \supseteq & \mathrm{PG}(U)
\end{array}
$$

It should be clear that any subset $T$ of $\mathbb{F}_{q}^{r n}$ or $\mathrm{PG}(r n-1, q)$ induces a subset in $\mathrm{PG}\left(r-1, q^{n}\right)$ in this way. In what follows we will use the same notation $\mathcal{B}(\pi)$ for the set of points of $\mathrm{PG}\left(r-1, q^{n}\right)$ induced by $\pi$, regardless of $\pi$ being a subspace or a subset of $\mathbb{F}_{q}^{r n}$ or $\operatorname{PG}(r n-1, q)$. Since the points of $\mathrm{PG}\left(r-1, q^{n}\right)$ correspond to 1-dimensional subspaces of $\mathbb{F}_{q^{n}}^{r}$, and by field reduction to $n$ dimensional subspaces of $\mathbb{F}_{q}^{r n}$, they correspond to a set $\mathcal{D}$ of $(n-1)$-dimensional subspaces of $\mathrm{PG}(r n-1, q)$, which partitions the pointset of $\mathrm{PG}(r n-1, q)$. The set $\mathcal{D}$ is called a Desarguesian spread, and we have a one-to-one correspondence between the points of $\mathrm{PG}\left(r-1, q^{n}\right)$ and the elements of $\mathcal{D}$. This gives us a more geometric perspective on the notion of a linear set; namely, an $\mathbb{F}_{q}$-linear set is a set $S$ of points of $\operatorname{PG}\left(r-1, q^{n}\right)$ for which there exists a subspace $\pi$ in $\mathrm{PG}(r n-1, q)$ such that the points of $S$ correspond to the elements of $\mathcal{D}$ that have a non-trivial intersection with $\pi$. Also in what follows, we will often identify the elements of $\mathcal{D}$ with the points of $\operatorname{PG}\left(r-1, q^{n}\right)$, which allows us to view $\mathcal{B}(\pi)$ as a subset of $\mathcal{D}$. This is illustrated by the following diagram, where $\mathcal{P}$ denotes the set of points of $\operatorname{PG}\left(r-1, q^{n}\right)$.

$$
\begin{aligned}
& \mathrm{PG}\left(r-1, q^{n}\right) \longleftrightarrow \mathrm{PG}(r n-1, q) \supseteq \pi \\
& \downarrow \quad \downarrow \quad \downarrow \\
& \mathcal{B}(\pi) \subseteq \mathcal{P} \quad \longleftrightarrow \quad \mathcal{D} \quad \supseteq \mathcal{B}(\pi)
\end{aligned}
$$


If $P$ is a point of $\mathcal{B}(\pi)$ in $\operatorname{PG}\left(r-1, q^{n}\right)$, where $\pi$ is a subspace of $\mathrm{PG}(r n-1, q)$, then we define the weight of $P$ as $w t(P):=\operatorname{dim}(P \cap \pi)+1$, where $P$ is identified with an element of $\mathcal{D}$. This makes a point have weight 1 if its corresponding spread element intersects $\pi$ in a point. Note that if $\pi=\mathrm{PG}(U)$, then $w t(P)=$ $\operatorname{dim}\langle\{v \in U \mid \operatorname{PG}(v)=P\}\rangle$. If $\alpha$ is a collineation of $\mathrm{PG}\left(r-1, q^{n}\right)$, then $\alpha$ is induced by a non-singular semi-linear map $\varphi$ of $V\left(r, q^{n}\right)$. Let $\left\langle v_{1}, \ldots, v_{w t(P)}\right\rangle$ be a basis for $\langle\{v \in U \mid \mathrm{PG}(v)=P\}\rangle$. The vectors $v_{i}^{\varphi}$ are linearly independent and clearly belong to $\left\langle\left\{v \in U^{\varphi} \mid \mathrm{PG}(v)=P^{\alpha}\right\}\right\rangle$, hence, $w t(P) \leq w t\left(P^{\alpha}\right)$. Repeating this argument for the collineation $\alpha^{-1}$ shows that $w t(P)=w t\left(P^{\alpha}\right)$, hence, a collineation preserves the weight of a point.

\section{Equivalent linear sets}

Two subsets $S$ and $T$ of $\operatorname{PG}(n, q)$ are called projectively equivalent if there is an element $\phi \in \operatorname{PGL}(n+1, q)$ such that $\phi(S)=T$, and equivalent if there is an element $\phi \in \operatorname{P\Gamma L}(n+1, q)$ such that $\phi(S)=T$.

The proofs in this section make use of yet another perspective on linear sets, namely as the quotient geometry of a canonical subgeometry. If $\mathcal{G}$ is a frame in $\mathrm{PG}\left(m, q^{n}\right)$, then the set of points (and subspaces) obtained by restricting the coordinates with respect to $\mathcal{G}$ to $\mathbb{F}_{q}$ is called a canonical subgeometry $\mathrm{PG}(m, q)$ of $\mathrm{PG}\left(m, q^{n}\right)$. It should be clear that a canonical subgeometry is a linear set, but not conversely. However, the following theorem by Lunardon and Polverino shows that every linear set is a projection of a canonical subgeometry. Let $\Sigma=\operatorname{PG}(m, q)$ be a canonical subgeometry of $\Sigma^{*}=\operatorname{PG}\left(m, q^{n}\right)$ and suppose there exists an $(m-r)$-dimensional subspace $\Omega^{*}$ of $\Sigma^{*}$ disjoint from $\Sigma$. Let $\Omega=\operatorname{PG}\left(r-1, q^{n}\right)$ be an $(r-1)$-dimensional subspace of $\Sigma^{*}$ disjoint from $\Omega^{*}$, and let $\Gamma$ be the projection of $\Sigma$ from $\Omega^{*}$ to $\Omega$. Let $p_{\Omega^{*}, \Omega}$ denote the map defined by $x \mapsto\left\langle\Omega^{*}, x\right\rangle \cap \Omega$ for each point $x \in \Sigma^{*} \backslash \Omega^{*}$.

Theorem 1. [10] If $\Gamma$ is a projection of $\mathrm{PG}(m, q)$ into $\Omega=\operatorname{PG}\left(r-1, q^{n}\right)$, then $\Gamma$ is an $\mathbb{F}_{q}$-linear set of rank $m+1$ and $\langle\Gamma\rangle=\Omega$. Conversely, if $L$ is an $\mathbb{F}_{q}$-linear set of $\Omega$ of rank $m+1$ and $\langle L\rangle=\Omega=\operatorname{PG}\left(r-1, q^{n}\right)$, then either $L$ is a canonical subgeometry of $\Omega$ or there are an $(m-r)$-dimensional subspace $\Omega^{*}$ of $\Sigma^{*}=\operatorname{PG}\left(m, q^{n}\right)$ disjoint from $\Omega$ and a canonical subgeometry $\Sigma$ of $\Sigma^{*}$ disjoint from $\Omega^{*}$ such that $L=p_{\Omega^{*}, \Omega}(\Sigma)$.

If we consider the quotientspace $\Sigma^{*} / \Omega^{*}$ instead of the projection we obtain the following.

Theorem 2. If $\Gamma$ is the quotient of $\mathrm{PG}(m, q)$ in $\Sigma^{*} / \Omega^{*} \cong \operatorname{PG}\left(r-1, q^{n}\right)$, then $\Gamma$ is an $\mathbb{F}_{q}$-linear set of rank $m+1$ and $\langle\Gamma\rangle=\Sigma^{*} / \Omega^{*}$. Conversely, if $L$ is an $\mathbb{F}_{q}$-linear set of rank $m+1$ and $\langle L\rangle=\mathrm{PG}\left(r-1, q^{n}\right)$, then there are an $(m-r)$ dimensional subspace $\Omega^{*}$ of $\Sigma^{*}=\mathrm{PG}\left(m, q^{n}\right)$ and a canonical subgeometry $\Sigma$ of $\Sigma^{*}$ disjoint from $\Omega^{*}$ such that $L$ is the quotient of $\Sigma$ in $\Sigma^{*} / \Omega^{*} \cong \operatorname{PG}\left(r-1, q^{n}\right)$.

Using this perspective on linear sets we derive the following equivalences which will be used in this section.

Theorem 3. Let $\pi_{1}, \pi_{2} \subset \mathrm{PG}(r n-1, q)$ and let $\mathcal{B}\left(\pi_{1}\right)$, respectively $\mathcal{B}\left(\pi_{2}\right)$, be a linear set of rank $m+1$ in $\mathrm{PG}\left(r-1, q^{n}\right)$, where $\mathcal{B}\left(\pi_{1}\right)$, respectively $\mathcal{B}\left(\pi_{2}\right)$, is the quotient of $\mathrm{PG}(m, q)$ in $\Sigma^{*} / \Omega_{1}^{*} \cong \mathrm{PG}\left(r-1, q^{n}\right)$, respectively the quotient of $\mathrm{PG}(m, q)$ in $\Sigma^{*} / \Omega_{2}^{*} \cong \operatorname{PG}\left(r-1, q^{n}\right)$. The following statements are equivalent. 
(i) There exists an element $\alpha \in \operatorname{P\Gamma L}\left(r, q^{n}\right)$ such that $\mathcal{B}\left(\pi_{1}\right)^{\alpha}=\mathcal{B}\left(\pi_{2}\right)$.

(ii) There exists an element $\beta \in$ Aut $\left(\Sigma^{*}\right) \cong \operatorname{P\Gamma L}\left(m+1, q^{n}\right)$ such that $\mathrm{PG}(m, q)^{\beta}=$ $\operatorname{PG}(m, q)$ and $\left(\Omega_{1}^{*}\right)^{\beta}=\Omega_{2}^{*}$.

Proof. Put $\Sigma:=\operatorname{PG}(m, q)$.

(ii) $\Rightarrow$ (i) Define $\alpha: \mathcal{B}\left(\pi_{1}\right) \mapsto \mathcal{B}\left(\pi_{2}\right),\left\langle\Omega_{1}^{*}, x\right\rangle / \Omega_{1}^{*} \mapsto\left\langle\Omega_{2}^{*}, x^{\beta}\right\rangle / \Omega_{2}^{*}$ with $x \in \Sigma$.

The map $\alpha$ is well defined: every element of $\mathcal{B}\left(\pi_{1}\right)$ can be written as $\left\langle\Omega_{1}^{*}, z\right\rangle / \Omega_{1}^{*}$ for some $z \in \Sigma$. Suppose that $\left\langle\Omega_{1}^{*}, z\right\rangle / \Omega_{1}^{*}=\left\langle\Omega_{1}^{*}, z^{\prime}\right\rangle / \Omega_{1}^{*}$ for some $z, z^{\prime}$ in $\Sigma$, then, since $\operatorname{dim}\left\langle z, z^{\prime}, \Omega_{1}^{*}\right\rangle=\operatorname{dim}\left\langle z^{\beta}, z^{\prime \beta},\left(\Omega_{1}^{*}\right)^{\beta}\right\rangle,\left(\left\langle\Omega_{1}^{*}, z\right\rangle / \Omega_{1}^{*}\right)^{\alpha}=\left(\left\langle\Omega_{1}^{*}, z^{\prime}\right\rangle / \Omega_{1}^{*}\right)^{\alpha}$. The map $\alpha$ is a collineation: if $\left\langle\Omega_{1}^{*}, z\right\rangle / \Omega_{1}^{*},\left\langle\Omega_{1}^{*}, z^{\prime}\right\rangle / \Omega_{1}^{*},\left\langle\Omega_{1}^{*}, z^{\prime \prime}\right\rangle / \Omega_{1}^{*}$ are collinear, then $\operatorname{dim}\left\langle z, z^{\prime}, z^{\prime \prime}, \Omega_{1}^{*}\right\rangle=m-r+2=\operatorname{dim}\left\langle z^{\beta}, z^{\prime \beta}, z^{\prime \prime \beta}, \Omega_{2}^{*}\right\rangle$, hence, $\left(\left\langle\Omega_{1}^{*}, z\right\rangle / \Omega_{1}^{*}\right)^{\alpha}$, $\left(\left\langle\Omega_{1}^{*}, z^{\prime}\right\rangle / \Omega_{1}^{*}\right)^{\alpha},\left(\left\langle\Omega_{1}^{*}, z^{\prime \prime}\right\rangle / \Omega_{1}^{*}\right)^{\alpha}$ are collinear. Moreover, $\mathcal{B}\left(\pi_{1}\right)^{\alpha}=\left(\left\langle\Omega_{1}^{*}, \Sigma\right\rangle / \Omega_{1}^{*}\right)^{\alpha}=$ $\left\langle\Omega_{2}^{*}, \Sigma\right\rangle / \Omega_{2}^{*}=\mathcal{B}\left(\pi_{2}\right)$

(i) $\Rightarrow$ (ii) Let $\tau_{i}, i=1,2$ be a subspace of $\Sigma^{*} \backslash \Omega_{i}^{*}$ and let $\Omega_{i}$, be an $(r-1)$ dimensional subspace, skew to $\Sigma$, and skew to $\Omega_{i}^{*}$. The projection of $\tau_{i}$ from $\Omega_{i}^{*}$ onto $\Omega_{i}$ is denoted by $p_{i}\left(\tau_{i}\right)$. Define for every point $x$ of $\Sigma$ the subspace $\sigma_{i}(x):=\left\langle\Omega_{i}^{*}, x\right\rangle \cap \Sigma$. We define a map $\gamma$ on the subspaces $\sigma_{1}(x)$ with $x \in \Sigma$ as $\sigma_{1}(x) \mapsto \sigma_{2}(y)$ if $p_{1}\left(\sigma_{1}(x)\right)^{\alpha}=p_{2}\left(\sigma_{2}(y)\right)$. The map $\gamma$ maps subspaces $\sigma_{1}(x)$ of dimension $d$ to subspaces $\sigma_{2}(y)$ of dimension $d$ since the weight of $p_{1}\left(\sigma_{1}(x)\right)$ is preserved by the collineation $\alpha$. We define a map $\delta$ on $\Sigma$ and show that $\delta$ is a collineation.

Assume first that there is a point $P$ in $\Sigma$ with $\sigma_{1}(P)=P$. Define $P^{\delta}:=P^{\gamma}$. Let $x \neq P$ be a point in $\Sigma$. Define $x^{\delta}:=\sigma_{1}(x)^{\gamma} \cap t$, where $t$ is the unique transversal line through $P^{\delta}$ to the set $\left\{\sigma_{1}(s)^{\gamma} \mid s \in P x\right\}$. Note that for all points $x$ with $\sigma_{1}(x)=x, x^{\delta}=x^{\gamma}$. Let $z \neq P$ be a point of $\Sigma$. Define $z^{\delta^{\prime}}=z^{\delta}$, and for all $x \neq$ $z \in \Sigma$ define $x^{\delta^{\prime}}:=\sigma_{1}(x)^{\gamma} \cap t$, where $t$ is the unique transversal line through $z^{\delta}$ to the set $\left\{\sigma_{1}(s)^{\gamma} \mid s \in z x\right\}$. We show that for all $y \in \Sigma, y^{\delta}=y^{\delta^{\prime}}$. Suppose that $y^{\delta} \neq$ $y^{\delta^{\prime}}$ and that $\operatorname{dim}\left\langle P^{\delta}, z^{\delta}, y^{\delta}, y^{\delta^{\prime}}\right\rangle=3$. The 3 -dimensional space $\left\langle P^{\delta}, z^{\delta}, y^{\delta}, y^{\delta^{\prime}}\right\rangle$ is projected onto the plane $\left\langle p_{2}\left(\sigma_{1}(y)^{\gamma}\right), p_{2}\left(P^{\delta}\right), p_{2}\left(\sigma_{1}(z)^{\gamma}\right)\right\rangle$. This is only possible if every point $p_{2}(z)$ with $z \in\left\langle P^{\delta}, z^{\delta}, y^{\delta}, y^{\delta^{\prime}}\right\rangle$ has weight 2 , a contradiction since $p_{2}(P)$ has weight one. Suppose that $y^{\delta} \neq y^{\delta^{\prime}}$ and that $\operatorname{dim}\left\langle P^{\delta}, z^{\delta}, y^{\delta}, y^{\delta^{\prime}}\right\rangle=2$. The line $y^{\delta^{\prime}} z^{\delta}$ and the line $P^{\delta} y^{\delta}$ meet in a point, hence, there is a subspace $\sigma_{1}(t)$ such that $P y$ meets $\sigma_{1}(t)$ in $t$ and $z y$ meets $\sigma_{1}(t)$ in $r$. The space $\left\langle P^{\delta}, z^{\delta}, r^{\delta}, t^{\delta}\right\rangle$ has dimension 3 since otherwise $\sigma_{2}\left(y^{\delta}\right)$ and $\sigma_{2}\left(t^{\delta}\right)$ would meet. Moreover it is projected from $\Omega_{2}^{*}$ onto a plane, again a contradiction since $p_{2}(P)$ has weight one. The map $\delta$ is a collineation: if $x, y, z$ are points on a line $L$ of $\Sigma$ through $P$, it is clear from the definition of $\delta$ that $x^{\delta}, y^{\delta}$ and $z^{\delta}$ are collinear. Let $x, y, z$ be collinear points on a line $L$ of $\Sigma$ not through $P$. In the previous part, we have showed that we can define $\delta$ as $x^{\delta}:=\sigma_{1}(x)^{\gamma} \cap t$, where $t$ is the unique transversal line through $w^{\delta}$ to the set $\left\{\sigma_{1}(s)^{\gamma} \mid s \in w x\right\}$ for some point $w$ of $L$. By this definition, it is clear that $x^{\delta}, y^{\delta}, z^{\delta}$ are collinear.

Next assume that there is no point $P$ in $\Sigma$ for which $\sigma_{1}(P)=P$ and let $P^{\prime}$ be a point in $\sigma_{1}(P)$. Choose a subspace $\Sigma^{\prime} \subset \Sigma$ for which $\sigma_{1}(P) \cap \Sigma^{\prime}=P$. In $\Sigma^{\prime}$, we can define a collineation $\tilde{\delta}$ as before. Define $P^{\delta}:=P^{\tilde{\delta}}$ and define $x^{\delta}$ for all $x \in \Sigma \backslash \sigma_{1}(P)$ as $\sigma_{1}(x)^{\gamma} \cap t$ where $t$ is the unique transversal line through $P^{\delta}$ to the set $\left\{\sigma_{1}(s)^{\gamma} \mid s \in P x\right\}$. Define $\left(P^{\prime}\right)^{\delta}, P^{\prime} \in \sigma_{1}(P)$ as $\sigma_{1}\left(P^{\prime}\right)^{\gamma} \cap t$ where $t$ is the unique transversal line through $z^{\delta}$ to the set $\left\{\sigma_{1}(s)^{\gamma} \mid s \in z x\right\}$, for some $z \notin \sigma_{1}(P)$. Let $\delta^{\prime}$ be the map defined by $x^{\delta^{\prime}}:=\sigma_{1}(x)^{\gamma} \cap t$, where $t$ is the unique transversal line through $P^{\prime \delta}$ to the set $\left\{\sigma_{1}(s)^{\gamma} \mid s \in P^{\prime} x\right\}$ and suppose 
that $y^{\delta} \neq y^{\delta^{\prime}}$ for some $y \notin \sigma_{1}(P)$. The 3 -dimensional space $\left\langle P^{\delta},\left(P^{\prime}\right)^{\delta}, y^{\delta}, y^{\delta^{\prime}}\right\rangle$ is mapped onto the line $\left\langle p_{2}\left(\sigma_{1}(P)^{\gamma}\right), p_{2}\left(\sigma_{1}(y)\right)^{\gamma}\right\rangle$, a contradiction since $p_{2}\left(\sigma_{1}(P)^{\gamma}\right)$ and $p_{2}\left(\sigma_{1}(y)\right)^{\gamma}$ have weight 2. The map $\delta$ is a collineation of $\Sigma$ : let $x, y, z$ be collinear points on the line $L$. If $L$ contains $P$, it is clear from the definition of $\delta$ that $x^{\delta}, y^{\delta}, z^{\delta}$ are collinear. If $\langle L, P\rangle \cap \sigma_{1}(P)=P, x^{\delta}=x^{\tilde{\delta}}, y^{\delta}=y^{\tilde{\delta}}, z^{\delta}=z^{\tilde{\delta}}$ are collinear since $\tilde{\delta}$ is a collineation. If $\langle L, P\rangle \cap \sigma_{1}(P)$ is a line $M$, there is a point $P^{\prime} \in \sigma_{1}(P)$ on $L$. Since the map $\delta$ defined by $P$ and the map $\delta^{\prime}$ defined by $P^{\prime}$ coincide on points outside of $\sigma_{1}(P), x^{\delta}, y^{\delta}, z^{\delta}$ are collinear. In the same way as the map $\delta$ defined by taking the intersection with the transversal line through $P^{\delta}$, the map $\delta$ defined by taking the intersections with the transversal lines through $z^{\delta}$ maps collinear points $x, y, z$, not in $\sigma_{1}(z)$, to collinear points. Hence, if $L \subset \sigma_{1}(P), x^{\delta}, y^{\delta}, z^{\delta}$ are collinear.

Since $\delta$ is a collineation of $\Sigma$, it corresponds to a semi-linear map $x \mapsto B x^{\theta}$, with $\theta \in \operatorname{Aut}\left(\mathbb{F}_{q}\right)$. The map $\alpha$ corresponds to a semi-linear map $x \mapsto A x^{\phi}$ with $\phi \in \operatorname{Aut}\left(\mathbb{F}_{q^{n}}\right)$. Let $x, y, z, w$ be collinear points of $\Sigma$. The cross ratio $\mu=$ $(x, y ; z, w) \in \mathbb{F}_{q}$ is preserved by projection, hence, $\mu=\left(p_{1}(x), p_{1}(y) ; p_{1}(z), p_{1}(w)\right)$. Moreover, $\left(x^{\delta}, y^{\delta} ; z^{\delta}, w^{\delta}\right)=\mu^{\theta}$ and $\left(p_{1}(x)^{\alpha}, p_{1}(y)^{\alpha} ; p_{1}(z)^{\alpha}, p_{1}(w)^{\alpha}\right)=\mu^{\sigma}$. But $\left(p_{2}\left(x^{\delta}\right), p_{2}\left(y^{\delta}\right) ; p_{2}\left(z^{\delta}\right), p_{2}\left(w^{\delta}\right)\right)=\left(p_{1}(x)^{\alpha}, p_{1}(y)^{\alpha} ; p_{1}(z)^{\alpha}, p_{1}(w)^{\alpha}\right)$. This shows that $\left.\theta\right|_{\mathbb{F}_{q}}=\left.\sigma\right|_{\mathbb{F}_{q}}$, hence, $\phi=\theta \psi$ with $\psi \in \operatorname{Aut}\left(\mathbb{F}_{q^{n}} / \mathbb{F}_{q}\right)$.

Now we define $\beta$ on $\Sigma^{*}$ as $x \mapsto B x^{\phi}$. Then, since $\psi$ fixes the points of $\Sigma$ pointwise, $\Sigma^{\beta}=\Sigma^{\delta}=\Sigma$, and since $\beta$ is a collineation, it maps the $(m-r)$-space $\Omega_{1}^{*}$ to an $(m-r)$-space. Since $\langle\Sigma\rangle=\Sigma^{*}$, a point $R$ of $\Omega_{1}$ can be written as a linear combination $\sum_{i=0}^{m} a_{i} x_{i}$, of points $x_{i}$ of $\Sigma$, with $a_{i} \in \mathbb{F}_{q^{n}}$. The point $R$ is mapped by $\beta$ to $R^{\beta}=\sum a_{i}^{\phi} x_{i}^{\beta}=\sum a_{i}^{\phi} y_{i}$ with $x_{i}^{\beta}=y_{i}$, hence, $p_{1}\left(x_{i}\right)^{\alpha}=p_{2}\left(y_{i}\right)$. Since $R=p_{1}(R), R^{\alpha}=\sum a_{i}^{\phi} p_{1}\left(x_{i}\right)^{\alpha}=\sum a_{i}^{\phi} p_{2}\left(y_{i}\right)$. This implies that $p_{2}\left(R^{\beta}\right)=R^{\alpha}$.

Define $o_{i}(\pi):=\left\langle\pi, \Omega_{i}^{*}\right\rangle \cap \Sigma$ for a subspace $\pi$ of $\Omega_{i}$. Now $\Omega_{1}^{*}=\cap\left\langle\Omega_{1}^{*}, \pi\right\rangle$, where the intersection is taken over all subspaces $\pi$ of $\Omega_{1}$ with $\operatorname{dim} o_{1}(\pi)=$ $\operatorname{dim} \Omega_{1}^{*}$. Suppose that there is a point $P \notin \Omega_{1}^{*}$, contained in this intersection, then the line through a point $Q$ in $\mathcal{B}\left(\pi_{1}\right) \in \Omega_{1}$ and $p_{1}(P)$ would meet $\Omega_{1}^{*}$, a contradiction. The space $\Omega_{1}^{* \beta}=\cap\left\langle\Omega_{1}^{*}, \pi\right\rangle^{\beta}=\cap\left\langle o_{1}(\pi), \pi\right\rangle^{\beta}=\cap\left\langle o_{1}(\pi)^{\beta}, \pi^{\beta}\right\rangle$, where the intersection is taken over all subspaces $\pi$ of $\Omega_{1}$ with $\operatorname{dim} o_{1}(\pi)=$ $\operatorname{dim} \Omega_{1}^{*}$. Since $\left\langle\Omega_{2}^{*}, \pi^{\beta}\right\rangle=\left\langle\Omega_{2}^{*}, \pi^{\alpha}\right\rangle$ and $o_{1}(\pi)^{\beta}=\left\langle\pi^{\alpha}, \Omega_{2}^{*}\right\rangle \cap \Sigma=o_{2}\left(\pi^{\alpha}\right)$, the image $\Omega_{1}^{* \beta}$ is equal to $\cap\left\langle\Omega_{2}^{*}, \pi^{\alpha}\right\rangle$, for all subspaces $\pi^{\alpha}$ of $\Omega_{2}$ with $\operatorname{dim} o_{2}\left(\pi^{\alpha}\right)=$ $\operatorname{dim} \Omega_{2}^{*}$. This shows that $\Omega_{1}^{* \beta}=\Omega_{2}^{*}$.

We turn our attention to the $\mathbb{F}_{q}$-linear sets of rank 3 in $\mathrm{PG}\left(1, q^{h}\right)$. Let $\mathcal{D}$ be the Desarguesian plane-spread in $\operatorname{PG}(2 h-1, q)$. Consider the linear set $\mathcal{B}(\pi)$, with $\pi \notin \mathcal{D}$ a plane of $\mathrm{PG}(2 h-1, q)$. If there is a spread element $H$ intersecting $\pi$ in a line, using the terminology introduced by Fancsali and Sziklai in [4], then $\mathcal{B}(\pi)$ is called a club and $H$ is called the head. If all elements of $\mathcal{D}$ intersecting $\pi$, intersect $\pi$ in a point, then $\mathcal{B}(\pi)$ is a scattered linear set of rank 3 .

In general, if all elements of $\mathcal{D}$ intersect a $k$-space $\mu$ in at most a point, then $\mu$ is called scattered with respect to $\mathcal{D}$ and $\mathcal{B}(\mu)$ is a scattered linear set of rank $k+1$ (see [2] for more on scattered spaces).

Remark 1.6 of [4] states without proof that a club in $\mathrm{PG}\left(1, q^{3}\right)$ is projectively equivalent to the set of points $\left\{x \in \mathbb{F}_{q^{3}} \| \operatorname{Tr}(x)=x+x^{q}+x^{q^{2}}=0\right\} \cup\{\infty\}$. In Corollary 5 , we show that indeed in the case $h=3$, all clubs of $\mathrm{PG}\left(1, q^{h}\right)$ are projectively equivalent, and that all scattered linear sets of rank 3 are projectively equivalent too. 
If $h>3$ however, the situation is different and linear sets of the same size are not necessarily (projectively) equivalent (see Theorem 5).

Lemma 4. Let $H \leq \operatorname{P\Gamma L}\left(3, q^{3}\right)$ denote the setwise stabiliser of a subplane $\pi \cong \mathrm{PG}(2, q)$ of $\mathrm{PG}\left(2, q^{3}\right)$, and put $H^{\prime}:=H \cap \mathrm{PGL}(3, q)$, let $T$ denote the set of points that do not lie on a secant line of $\pi$, and let $S$ denote the set of points of $\mathrm{PG}\left(2, q^{3}\right) \backslash \pi$ that lie on a secant line of $\pi$.

(i) For each point $X$ of $\pi$, the stabiliser $H_{X}^{\prime}$ of $X$ in $H^{\prime}$ acts sharply transitive on the set $T$, and for each point $R$ of $T$, the stabiliser $H_{R}^{\prime}$ of $R$ in $H^{\prime}$ acts transitively on the points of $\pi$.

(ii) The group $H^{\prime}$ acts transitively on the set $S$. The stabiliser $H_{Z}^{\prime}$ of a point $Z \in S$ acts transitively on the points of $\pi$ not lying on the secant line through $Z$.

Proof. (i) The set $T$ has size

$$
t:=q^{6}+q^{3}+1-\left(q^{2}+q+1\right)-\left(q^{2}+q+1\right)\left(q^{3}-q\right)=q^{6}-q^{5}-q^{4}+q^{3} .
$$

Let $X \in \pi$. Since $H^{\prime}$ acts transitively on the points of $\pi$ and has size $\left(q^{2}+q+1\right) t$, we have $\left|H_{X}^{\prime}\right|=t$. We show that $H_{X}^{\prime}$ acts sharply transitive on the points of $T$, by proving that $\left|H_{X Y}^{\prime}\right|$ is trivial, for each $Y \in T$. An element of $H_{X Y}^{\prime}$ corresponds to a matrix $A$ with entries in $\mathbb{F}_{q}$ with an eigenvalue in $\mathbb{F}_{q}$, with eigenvector $X$, and an eigenvalue in $\mathbb{F}_{q^{3}} \backslash \mathbb{F}_{q}$, corresponding to $Y$. But $Y^{q}$ and $Y^{q^{2}}$, are also fixed by $A$. Since $Y \in T, Y, Y^{q}$ and $Y^{q^{2}}$ are linearly independent. Since a matrix $A$ can have at most three eigenvalues which correspond to linearly independent points, and there are already three linearly independent points with eigenvalue in $\mathbb{F}_{q^{3}} \backslash \mathbb{F}_{q}$, there cannot be an eigenvector with eigenvalue in $\mathbb{F}_{q}$. This implies that $H_{X Y}^{\prime}$ is trivial.

For each point $R \in T$, there exists an element $\alpha \in \operatorname{PGL}(3, q)$ of order $q^{2}+q+1$ (generating a Singer cycle) that fixes $R$. This implies that the size of $H_{R}^{\prime}$ is at least $q^{2}+q+1$. Since the stabiliser $H_{R X}^{\prime}$ of a point $X \in \pi$ is trivial, and the orbit of a point of $\pi$ can have length at most $q^{2}+q+1$, from $\left|H_{R}^{\prime}\right|=\left|H_{R X}^{\prime}\right|\left|X^{H_{R}^{\prime}}\right|$, we derive that $\left|X^{H_{R}^{\prime}}\right|=q^{2}+q+1$. So $H_{R}^{\prime}$ acts transitively on the points of $\pi$.

(ii) The number of points in $S$ is equal to

$$
s:=\left(q^{2}+q+1\right)\left(q^{3}-q\right) .
$$

Let $Z$ be a point of $S$, and let $L$ be the secant line to $\operatorname{PG}(2, q)$ through $Z$. Since an element of $H_{Z}^{\prime}$ fixes three different points $Z, Z^{q}, Z^{q^{2}}$ on $L$, it must fix $L$ pointwise. It follows that an element of $H_{Z X}^{\prime}$, with $X \in \pi \backslash L$, is a homology with centre $X$ and axis $L$, and each homology with center $X$ and axis $L$ clearly belongs to $H_{Z X}^{\prime}$. It follows that $\left|H_{Z X}^{\prime}\right|=q-1$. Since the group of elations of $\pi$ with axis $L$ acts transitively on the points not on $L$, $\left|X^{H_{Z}^{\prime}}\right|=q^{2}$. Now $\left|H_{Z}^{\prime}\right|=\left|H_{Z X}^{\prime}\right|\left|X^{H_{Z}^{\prime}}\right|=(q-1) q^{2},\left|H^{\prime}\right|=\left|H_{Z}^{\prime}\right|\left|Z^{H^{\prime}}\right|$ and $\left|H^{\prime}\right|=\left(q^{2}+q+1\right)\left(q^{6}-q^{5}-q^{4}+q^{3}\right)$, hence $\left|Z^{H^{\prime}}\right|=\left(q^{2}+q+1\right)\left(q^{3}-q\right)=s$. This implies that $H^{\prime}$ acts transitively on the points of $U$.

Theorem 5. (i) All clubs in $\mathrm{PG}\left(1, q^{3}\right)$ and all scattered linear spaces in $\mathrm{PG}\left(1, q^{3}\right)$ are projectively equivalent. 
(ii) All clubs and all scattered linear sets of rank 3 in $\mathrm{PG}\left(1,2^{5}\right)$ are equivalent, but there exist projectively inequivalent clubs and projectively inequivalent scattered linear spaces of rank 3 in $\mathrm{PG}\left(1,2^{5}\right)$.

(iii) There exist inequivalent clubs and inequivalent scattered linear sets of rank 3 in $\mathrm{PG}\left(1, q^{h}\right)$ with $h \neq 3$ and $(h, q) \neq(5,2)$.

Proof. Let $H$ be the setwise stabiliser in $\mathrm{P} \Gamma \mathrm{L}\left(3, q^{h}\right)$ of a subplane $\pi \cong \mathrm{PG}(2, q)$ of $\mathrm{PG}\left(2, q^{h}\right)$. Let $T$ denote the set of points that do not lie on a secant line of $\pi$, and let $S$ denote the set of points of $\mathrm{PG}\left(2, q^{3}\right) \backslash \pi$ that lie on a secant line of $\pi$. By the equivalent perspective on linear sets using quotient geometries and Theorem 3 it suffices to study the transitivity of the action of $H$ on the sets $T$ and $S$. Since the group PGL $(3, q)$ acts sharply transitive on the frames of $\operatorname{PG}(2, q)$ and the only element of $\operatorname{PGL}\left(3, q^{h}\right)$ fixing a frame is the identity element, it follows that

$$
|H|=h|\operatorname{P\Gamma L}(3, q)|=h k q^{3}\left(q^{3}-1\right)\left(q^{2}-1\right),
$$

where $q=p^{k}, p$ prime. Calculating the size of $T$ and $S$ we get

$$
|T|=q^{2 h}-q^{h+2}-q^{h+1}+q^{3} \text { and }|S|=q^{h+2}+q^{h+1}+q^{h}-q^{3}-q^{2}-q .
$$

Using Theorem 3 it follows that there are inequivalent scattered linear sets of rank 3 in $\mathrm{PG}\left(1, q^{h}\right), h \geq 6$ and in $\mathrm{PG}\left(1, q^{5}\right)$, for $q>2$, and that there are inequivalent clubs in $\mathrm{PG}\left(1, q^{h}\right), h>7$, and in $\mathrm{PG}\left(1, q^{7}\right)$ for $q>5$. If $H$ acts transitively on $S$, then $|S|$ has to divide $|H|$. If $h=5$, this yields that $p^{2 k}+1$ has to divide $5 k\left(p^{k}-1\right)$. This is only possible in the cases $k=1, p=2,3$. If $h=7$, this argument yields that $p^{4 k}+p^{2 k}+1$ has to divide $7 k\left(p^{3 k}-p^{k}\right)$, which is not possible. If $h$ is not a prime, then by the induced action of $H$ on subplanes containing $\pi$, it follows that $H$ does not act transitively on $S$ neither on $T$. If $h=3$, then $H$ acts transitively on both $T$ and $S$ by Lemma 4 . This leaves only the cases $\mathrm{PG}\left(2, q^{5}\right)$ with $q=2,3$. Let $Z$ be a point of $S$ on a secant $L$ of $\pi$. Since $H_{Z}$ fixes $Z, Z^{q}, Z^{q^{2}}, Z^{q^{3}}, Z^{q^{4}}$ on $L, L$ is fixed pointwise. The elements of $H_{Z}$ consist of an element $\phi$ of PGL $(3, q)$ and an element $\alpha$ of $A u t\left(\mathbb{F}_{q^{h}}\right)$ and since $Z$ is fixed pointwise, $\alpha$ is trivial and $H_{Z}=H_{Z}^{\prime}$, with $H^{\prime}=\operatorname{PGL}(3, q)$. As in the proof of Lemma 4, one shows that the size of $H_{Z}^{\prime}$ is equal to $(q-1) q^{2}$. If $q^{h}=2^{5}$, then $|H|=840=\left|H_{Z}\right| \cdot\left|Z^{H}\right|=4 .\left|Z^{H}\right|$. Since $|S|=210, H$ acts transitively on the points of $S$. Together with Theorem 3 this shows that all clubs in $\mathrm{PG}\left(1,2^{5}\right)$ are equivalent. Since $\left|H_{Z}\right|=\left|H_{Z}^{\prime}\right|=4$ and $\left|H^{\prime}\right|=168,\left|Z^{H^{\prime}}\right|=42<210$, hence, not all clubs in $\mathrm{PG}\left(1,2^{5}\right)$ are projectively equivalent.

If $q=3^{5}$, then $\left|H_{Z}\right|=18$ and $|H|=28080$, from which it follows that $\left|Z^{H}\right|=1560<|S|=3120$, which implies that there are inequivalent clubs in $\mathrm{PG}\left(1,3^{5}\right)$.

Let $X$ be a point if $T$ in $\mathrm{PG}\left(2,2^{5}\right)$. An element of $H_{X}$ corresponds to a $3 \times 3$ matrix $A$ with entries in $\mathbb{F}_{q}$, having 5 eigenvectors, 3 of which are independent, each corresponding to an eigenvalue of $A$ in $\mathbb{F}_{2^{5}} \backslash \mathbb{F}_{2}$, a contradiction unless $A$ is the identity matrix. Hence, $\left|H_{X}\right|=1$ and $\left|X^{H}\right|=|H|=840=|T|$. So $H$ acts transitively on the points of $T$, and by Theorem 3 all scattered linear sets of rank 3 in $\mathrm{PG}\left(1,2^{5}\right)$ are equivalent. Since $H_{X}^{\prime}<H_{X}$ and $H_{X}$ is trivial, $\left|H^{\prime}\right|=168=\left|X^{H^{\prime}}\right|<840$ hence, there are scattered linear sets of rank 3 in $\mathrm{PG}\left(1,2^{5}\right)$ that are projectively inequivalent. 


\section{The intersection of a subline and an $\mathbb{F}_{q}$-linear set in $\mathrm{PG}\left(1, q^{h}\right)$}

The intersection of a subline and a club of $\mathrm{PG}\left(1, q^{h}\right)$ was already investigated in [4]. However, in this proof, the authors use that all clubs of $\mathrm{PG}\left(1, q^{h}\right)$ are projectively equivalent, which is in general not true (see Corollary 5 ). Theorem 8 shows that their result is correct and Lemma 6 gives a description of the geometric structure of the intersection points of the subline and the linear set. Consider a subline $\ell \cong \operatorname{PG}(1, q)$ of $\mathrm{PG}\left(1, q^{h}\right)$. Using the notation introduced above, $\ell$ is an $\mathbb{F}_{q}$-linear set of $\mathrm{PG}\left(1, q^{h}\right)$ of rank two. In the spread representation $\ell=\mathcal{B}(L)$, where $L$ is a line in $\operatorname{PG}(2 h-1, q)$ not contained in an element of $\mathcal{D}$, i.e. an $\mathbb{F}_{q}$-subline $\ell$ of $\operatorname{PG}\left(1, q^{h}\right)$ corresponds to an $(h-1)$-regulus $\mathcal{B}(L)$ in $\mathrm{PG}(2 h-1, q)$.

Lemma 6. Let $\mathcal{R}=\left\{\sigma_{1}, \ldots, \sigma_{q+1}\right\}$ be an $(h-1)$-regulus in $\mathrm{PG}(2 h-1, q)$, and let $\pi$ be a plane in $\mathrm{PG}(2 h-1, q)$ such that $\pi \cap \sigma_{i}$ is a point $P_{i}, i=1, \ldots, 4$, where no three points of $\left\{P_{1}, P_{2}, P_{3}, P_{4}\right\}$ are collinear. Then $\pi \cap \sigma_{i}$ is a point $P_{i}$ for all $1 \leq i \leq q+1$ and $\left\{P_{1}, \ldots, P_{q+1}\right\}$ are the points of a conic in $\pi$.

Proof. Let $\mathcal{R}=\left\{\sigma_{1}, \ldots, \sigma_{q+1}\right\}$ be a regulus, and let $\pi$ be a plane such that $\pi \cap \sigma_{i}$ is a point $P_{i}, i=1, \ldots, 4$, where no three points of $\left\{P_{1}, P_{2}, P_{3}, P_{4}\right\}$ are collinear. Let $t$ be the transversal line to $\mathcal{R}$ through $P_{1}$. Let $P_{j}^{\prime}$ be the points $\sigma_{j} \cap t, j=2,3,4$. The 3 -dimensional space $\langle t, \pi\rangle$ intersects $\sigma_{j}$ in the line $\ell_{j}=P_{j} P_{j}^{\prime}, j=2,3,4$. Hence, $t$ is a transversal line to the 1 -regulus $\mathcal{R}\left(\ell_{2}, \ell_{3}, \ell_{4}\right)$. The transversal line $t^{\prime}$ through $P_{2}$ to the regulus $\mathcal{R}\left(\ell_{2}, \ell_{3}, \ell_{4}\right)$ intersects the spreadelements $\sigma_{i}$ in points $P_{i}, i=5, \ldots, q+1$, and intersects $\sigma_{1}$, in the point $P_{1}^{\prime}$. This implies that the line $P_{i}^{\prime} P_{i}$, contained in $\sigma_{i}, i=1, \ldots, q+1$, is a line of the regulus $\mathcal{R}\left(\ell_{2}, \ell_{3}, \ell_{4}\right)$. Hence, the elements of $\mathcal{R}$ intersect the 3 -dimensional space $\langle t, \pi\rangle$ in the lines of a regulus of a hyperbolic quadric. Since $\pi$ is a plane of $\langle t, \pi\rangle$, not containing a line of an element of $\mathcal{R},\left\{P_{1}, \ldots, P_{q+1}\right\}$ are the points of a conic.

Lemma 7. If a $(2 k-1)$-space $\pi$ intersects 3 elements of an $(h-1)$-regulus in $a(k-1)$-dimensional subspace, it intersects $\pi$ in a $(k-1)$-regulus.

Proof. Let $\sigma_{i}$, with $i=1,2,3$, be the 3 elements of $\mathcal{B}(L)$, with $\mathcal{B}(L)$ an $(h-1)$ regulus, intersecting some $(2 k-1)$-space $\pi$ in a $(k-1)$-space $S_{i}$. The spaces $S_{1}, S_{2}, S_{3}$ determine a unique $(k-1)$-regulus $\mathcal{R}$. Let $S_{k}$ be an element of $\mathcal{R}$. A transversal line $t$ through a point $P$ of $S_{k}$ to $\mathcal{R}$ intersects the elements $\sigma_{i}, i=$ $1,2,3$ in a point of $S_{i}$. Hence, $t$ is the unique transversal line through $P$ to $\mathcal{B}(L)$ and it follows that every element of the regulus through $S_{1}, S_{2}, S_{3}$ is contained in an element of the regulus $\mathcal{B}(L)$, and conversely every element of $\mathcal{B}(L)$ contains an element of the regulus through $S_{1}, S_{2}, S_{3}$.

Theorem 8. A subline $\mathrm{PG}(1, q)$ intersects an $\mathbb{F}_{q}$-linear set of rank $k$ of $\mathrm{PG}\left(1, q^{h}\right)$ in $0,1, \ldots, k$ or $q+1$ points.

Proof. We proceed by induction on the rank $k$. For $k=2$, the theorem follows from the observation that 3 points determine a unique subline. So now suppose $k>2$ and assume that the statement holds for $k^{\prime}<k$. Let $\pi$ be a $(k-1)$ dimensional space. Let $\mathcal{B}\left(L_{1}\right)$ be a subline of $\mathrm{PG}\left(1, q^{h}\right)$, intersecting $\mathcal{B}(\pi)$ in 
at least $k+1$ points. Let $\sigma_{1}, \ldots, \sigma_{k+1}$ be elements of $\mathcal{B}\left(L_{1}\right)$, intersecting $\pi$. We may choose $L_{1}$ to go through a point $R_{1}$ of $\sigma_{1} \cap \pi$. Let $R_{i}$ be a point in $\sigma_{i} \cap \pi$. If one of the intersections $\sigma_{i} \cap \pi$, say $\sigma_{2} \cap \pi$ contains a line $M$, then the space $\mu=\left\langle R_{1}, R_{3}, \ldots, \ldots, R_{k}\right\rangle$ intersects $M$ in a point, and we have that $\mathcal{B}\left(L_{1}\right)$ intersects $\mathcal{B}(\mu)$, which has rank $k-1$, in $k+1$ points. By induction, $\mathcal{B}\left(L_{1}\right)$ is contained in $\mathcal{B}(\mu) \subset \mathcal{B}(\pi)$.

So from now on, we assume that all intersections $\sigma_{i} \cap \pi, i=1, \ldots, k+1$, are points $R_{i}$. Suppose that $r+1$ points of $\left\{R_{1}, \ldots, R_{k+1}\right\}$ are contained in an $r$-1-dimensional subspace $\nu$ of $\pi, r<k$, then again by induction on $k, \mathcal{B}(L)$ is contained in $\mathcal{B}(\nu) \subset \mathcal{B}(\pi)$.

Hence, from now on, we also assume that no $r+1$ points of $\left\{R_{1}, \ldots, R_{k+1}\right\}$ are contained in an $(r-1)$-dimensional space, $r<k$.

Let $L_{i}$ be the transversal line through $R_{i}, i=2, \ldots, k-2$, to the regulus $\mathcal{B}\left(L_{1}\right)$.

Let $\phi_{i}$ be the space $\left\langle L_{1} \cap \sigma_{i}, \ldots, L_{k-2} \cap \sigma_{i}\right\rangle$, with $\operatorname{dim} \phi_{i}=k-3-x$, for all $1 \leq i \leq q+1$. The $(k-3)$-space $\psi=\left\langle R_{1}, \ldots, R_{k-2}\right\rangle$ is contained in the $(2 k-2 x-5)$-space $\left\langle\phi_{1}, \phi_{2}\right\rangle$. Hence, if $x>0, \phi_{i} \subset \sigma_{i}$ meets $\psi \subset \pi$ for all $i \leq q+1$, so, $\mathcal{B}(L) \subset \mathcal{B}(\pi)$.

Assume that $\operatorname{dim} \phi_{i}=k-3$. If one of the points $R_{j} \in \phi_{j}$ for some $j \in$ $\{k-1, k, k+1\}$, then $\operatorname{dim}\left\langle\pi, L_{1} \ldots, L_{k-2}\right\rangle \leq 2 k-4$, and hence $\pi$ intersects each $\phi_{i}$, i.e., $\mathcal{B}(L) \subset \mathcal{B}(\pi)$.

If $R_{j} \notin \phi_{j}$ for all $j \in\{k-1, k, k+1\}$, the $(k-2)$-spaces $\left\langle R_{i}, \phi_{i}\right\rangle, i=$ $k-1, k, k+1$, contained in the $(2 k-3)$-space $\nu=\left\langle\pi, L_{1}, \ldots, L_{k-2}\right\rangle$, determine a $(k-2)$-regulus $\left\{\tau_{1}, \ldots, \tau_{q+1}\right\}$, by Lemma 7 . Since for all $1 \leq i \leq q+1$, the $(k-2)$-space $\tau_{i} \subset \sigma_{i}$ and the $(k-1)$-space $\pi$, contained in the $(2 k-3)$-space $\nu$, intersect in a point, $\mathcal{B}\left(L_{1}\right) \subset \mathcal{B}(\pi)$.

Theorem 9. For every subline $L \cong \mathrm{PG}(1, q)$ of $\mathrm{PG}\left(1, q^{h}\right)$, there is a linear set $S$ of rank $k, k \leq h$, intersecting $L$ in exactly $j$ points, for all $1 \leq j \leq k$.

Proof. The statement is trivial for $j \in\{1,2\}$, so fix $3 \leq j \leq k$. Let $\mathcal{B}(L)=$ $\left\{\sigma_{1}, \ldots, \sigma_{q+1}\right\}$ be a subline of $\mathrm{PG}\left(1, q^{h}\right)$. Let $t_{P}$ denote the transversal line to $\mathcal{B}(L)$ through a point $P$. Let $P_{1}$ be the point $\sigma_{1} \cap L$, let $P_{i}$ be a point in $\sigma_{i} \backslash\left\langle t_{P_{1}}, \ldots, t_{P_{i-1}}\right\rangle$, for $2 \leq i \leq j-1$, and let $\pi_{j}$ be a $(k-j)$-space in $\sigma_{j} \backslash\left\langle t_{P_{1}}, \ldots, t_{P_{j-1}}\right\rangle$. Let $\pi$ be the space $\left\langle P_{1}, \ldots, P_{j-1}, \pi_{j}\right\rangle$, hence $\mathcal{B}(\pi)$ is a linear set of rank $k$. We show that $\mathcal{B}(\pi)=\left\{\sigma_{1}, \ldots, \sigma_{j}\right\}$.

Suppose that one extra element of $\mathcal{B}(L)$, say $\sigma_{j+1}$, is contained in $\mathcal{B}(\pi)$ and let $P_{j+1}$ be a point in the intersection of $\sigma_{j+1}$ with $\pi$. The $j-1$-space $\mu=\left\langle P_{1}, \ldots, P_{j-1}, P_{j+1}\right\rangle$ meets the $k-j$-space $\pi_{j}$, both contained in the $k-1$ space $\pi$ in the point $P_{j+1}$. Hence, the $j-1$-space $\mu$ meets $j+1$ elements of $\left\{\sigma_{1}, \ldots, \sigma_{q+1}\right\}$, and it follows from Theorem 8 that $\mathcal{B}(L)$ is contained in $\mathcal{B}(\mu)$.

The intersection of the $(2 j-3)$-space $\left\langle\mu, t_{P_{1}}, t_{P_{2}}, \ldots, t_{P_{j-2}}\right\rangle$ with the elements $\sigma_{i}$ forms a $(j-2)$-regulus. Hence, the transversal line $T$ through $P_{j-1}$ to this regulus meets $\sigma_{j}$ in a point $Q$. Since $T$ is a transversal to $\mathcal{B}(L)$ through $P_{j-1}$, $Q$ has to be the intersection point of $t_{P_{j-1}}$ with $\sigma_{j}$, a contradiction.

\section{Sublines contained in a linear set}

Throughout this section, we let $\mathcal{D}=\left\{\sigma_{1}, \ldots, \sigma_{q^{h}+1}\right\}$ denote the Desarguesian $(h-1)$-spread of PG $(2 h-1, q)$ and by a subline, we mean an $\mathbb{F}_{q}$-subline PG $(1, q)$. 
If $\pi$ is a subspace of $\mathrm{PG}(2 h-1, q)$ and $s$ and $r$ are points of $\pi$, then the subline $\mathcal{B}(r s)$ is clearly contained in $\mathcal{B}(\pi)$. In this section, we investigate the possibility of other sublines through $\mathcal{B}(r)$ and $\mathcal{B}(s)$, contained in $\mathcal{B}(\pi)$. A subline $\mathcal{B}(L)$ of $\mathcal{B}(\pi)$ is called irregular if there is no line $M$ of $\pi$ such that $\mathcal{B}(M)=\mathcal{B}(L)$.

Lemma 10. Let $\pi$ be a 3 - dimensional space in $\mathrm{PG}(2 h-1, q)$. The intersection of the elements of $\mathcal{D}$ with $\pi$ is one of the following:

1. $\pi$ is contained in an element of $\mathcal{D}$

2. $\pi$ is scattered with respect to $\mathcal{D}$

3. one element of $\mathcal{D}$ intersects $\pi$ in a plane, $q^{3}$ elements of $\mathcal{D}$ intersect $\pi$ in a point

4. one or two elements of $\mathcal{D}$ intersect $\pi$ in a line and the other elements of $\mathcal{D}$ that intersect $\pi$, intersect $\pi$ in a point

5. $q+1$ elements of $\mathcal{D}$ intersect $\pi$ in a line, $q^{3}-q$ elements of $\mathcal{D}$ intersect $\pi$ in a point. In this case, the $q+1$ lines that are the intersection of an element of $\mathcal{D}$ with $\pi$ form a (line-)regulus in $\pi$

6. all elements of $\mathcal{D}$, intersecting $\pi$, intersect $\pi$ in a line and in this case, the elements intersecting $\pi$ form a $\mathrm{PG}\left(1, q^{2}\right)$.

Moreover, if $h$ is odd, possibility 6 cannot occur and if $h=3$, only the possibilities 3 and 5 occur.

Proof. If $\pi$ contains a plane of $\mathcal{D}$, it is clear that all other intersections of an element of $\mathcal{D}$ with $\pi$ are points. Suppose now that only lines and points occur as intersection of an element of $\mathcal{D}$ with $\pi$. Let $L_{1}, L_{2}, L_{3}$ be three lines in $\pi$ that occur as intersection of $\sigma_{1}, \sigma_{2}, \sigma_{3} \in \mathcal{D}$ with $\pi$ and let $t$ be a transversal line to $L_{1}, L_{2}, L_{3}$, which exists since the lines $L_{i}$ are contained in a 3 -dimensional space. The line $t$ is a transversal line to $\sigma_{1}, \sigma_{2}, \sigma_{3}$, hence, intersects $q-2$ other spread elements of $\mathcal{D}$, say $\sigma_{4}, \ldots, \sigma_{q+1}$. A transversal line $t^{\prime} \neq t$ to $\mathcal{B}(t)$ intersects $\sigma_{1}, \sigma_{2}, \sigma_{3}$, hence, also $\sigma_{4}, \ldots, \sigma_{q+1}$, which implies that the intersection of $\sigma_{i}$ with $\pi$ is a line $L_{i}$, and that the lines $L_{i}$ form a regulus. Suppose now that there is a line $M \neq L_{i}$ contained in $\pi$, with $\mathcal{B}(M) \cap \pi=M$. Since the regulus through three of the lines $\left\{L_{1}, \ldots, L_{q+1}, M\right\}$ is contained in $\pi$, we easily see that in that case, every element of $\mathcal{D}$ intersects $\pi$ in a line. The $q^{2}+1$ elements of $\mathcal{D}$ intersecting $\pi$ in the Desarguesian linespread form a $\operatorname{PG}\left(1, q^{2}\right)$, embedded in $\operatorname{PG}\left(1, q^{h}\right)$, hence, $h$ is even.

Let $A$ be the number of elements of $\mathcal{D}$ intersecting $\pi$ in a line, and suppose that no element of $\mathcal{D}$ intersects $\pi$ in a line. If $h=3$, then $A(q+1)+\left(q^{3}+1-A\right)=$ $q^{3}+q^{2}+q+1$ since all $q^{3}+1$ elements of the plane spread $\mathcal{D}$ in $\operatorname{PG}(5, q)$ intersect the 3 -dimensional space $\pi$. If follows that $A=q+1$.

\subsection{Sublines contained in a club}

In this subsection, we show that there are no irregular sublines contained in a club $S \neq \mathrm{PG}\left(1, q^{2}\right)$.

Lemma 11. If $S \cong \mathrm{PG}\left(1, q^{2}\right)$, then there are exactly $q+1$ different sublines through two points of $S$, contained in $S$. 
Proof. If $S=\mathcal{B}(\pi) \cong \operatorname{PG}\left(1, q^{2}\right)$, then there is a 3 -dimensional space $\mu$ through $\pi$ such that $\mathcal{D}$ intersects $\mu$ in $q^{2}+1$ lines. Let $\mathcal{B}(r)$ and $\mathcal{B}(s)$ be two different points of $S$, with $r, s \in \pi$, and let $\mathcal{B}(s) \cap \mu$ be the line $L$. Any of the $q+1$ lines $t_{i}$ through $r$ and a point of $L$ intersects $\mathcal{D}$ in $q+1$ elements of $\mathcal{B}(S)$, hence, $\mathcal{B}\left(t_{i}\right)$ is contained in $S$. If $\mathcal{B}\left(t_{i}\right)=\mathcal{B}\left(t_{j}\right)$ for some $i \neq j$, then $t_{i}=t_{j}$ since $t_{i}$ and $t_{j}$ are transversal lines to the regulus $\mathcal{B}\left(t_{i}\right)$ through the point $r$.

Lemma 12. Let $\pi$ be a plane in $\mathrm{PG}(2 h-1, q)$, such that $\mathcal{B}(\pi) \neq \mathrm{PG}\left(1, q^{2}\right)$. If there is an irregular subline $\mathcal{B}(L)$ contained in $\mathcal{B}(\pi)$, then $\pi$ is scattered with respect to $\mathcal{D}$.

Proof. Suppose that $\pi$ is a plane such that $\mathcal{B}(\pi) \neq \mathrm{PG}\left(1, q^{2}\right)$ and that $L$ is a line, intersecting $\pi$ in exactly one point, such that $\mathcal{B}(L) \subset \mathcal{B}(\pi)$. This implies that at least $q+1$ elements of $\mathcal{D}$ intersect $\langle\pi, L\rangle$ in a line. But then there are exactly $q+1$ elements of $\mathcal{D}$ intersecting $\mu$ in a line (see Lemma 10), where the $q+1$ intersection lines form a regulus $\mathcal{R}=\left\{L_{1}, \ldots, L_{q+1}\right\}$. Suppose that there is a line $L_{i}$ of $\mathcal{R}$ contained in $\pi$, then $P=\pi \cap L \in L_{i}$. The plane $\pi$ contains one line $L_{i}$ of a regulus $\mathcal{R}$, hence, it has to contain a transversal line to $\mathcal{R}$ through $P$, a contradiction since $L \notin \pi$ is the transversal line through $P$ to $\mathcal{R}$.

Hence, no line of $\mathcal{R}$ is contained in $\pi$, so $\pi$ is scattered.

If $\mathcal{B}(\pi)$ is a club $¥ \mathrm{PG}\left(1, q^{2}\right)$ with head $H$ and $\mathcal{B}(r)$ and $\mathcal{B}(s), r, s \in \pi$ are two non-head points, then the line $r s$ meets $H$ in a point $p$. Hence, the subline $\mathcal{B}(r s)$ through $\mathcal{B}(r)$ and $\mathcal{B}(s)$ always contains the head $H$. As a corollary to the previous lemma, we show that every subline contained in $\mathcal{B}(\pi)$ is regular and contains the head.

Corollary 13. If $S$ is a club of $\mathrm{PG}\left(1, q^{h}\right)$, where $S \neq \mathrm{PG}\left(1, q^{2}\right)$, then there are no irregular sublines contained in $S$. Hence, through 2 non-head points of a club $S \neq \mathrm{PG}\left(1, q^{2}\right)$ of $\mathrm{PG}\left(1, q^{h}\right)$, there is exactly one subline contained in $S$, which contains the head of the club.

\subsection{Sublines contained in a scattered linear set of rank 3}

In this subsection, we show that there are irregular sublines contained in a linear set of rank 3 .

Lemma 14. Let $\pi$ be a plane in $\mathrm{PG}(2 h-1, q)$, let $\mathcal{B}(r)$ and $\mathcal{B}(s)$ be two different points of $\mathcal{B}(\pi)$, with $r, s \in \pi$. Then the following statements hold.

1. There is exactly one 3 -dimensional space $\mu$ through $\pi$ such that $\mu$ intersects $\mathcal{B}(r)$ and $\mathcal{B}(s)$ in a line.

2. If there is a line $L$ through $r, L \notin \pi$, such that $\mathcal{B}(s) \in \mathcal{B}(L)$ and $\mathcal{B}(L)$ is contained in $\pi$, then $\langle\pi, L\rangle$ intersects $\mathcal{B}(s)$ and $\mathcal{B}(r)$ in a line.

Proof. (1) Let $\pi$ be a plane in $\mathrm{PG}(2 h-1, q)$, let $\mathcal{B}(r)$ and $\mathcal{B}(s)$ be two different points of $\mathcal{B}(\pi)$, with $r, s \in \pi$. Since $\langle\mathcal{B}(s), \pi\rangle$ is a $(h+1)$-space, it intersects the $(h-1)$-space $\mathcal{B}(r)$ in a subspace $L_{r}$ of dimension at least 1 . It is not possible that $\langle\mathcal{B}(s), \pi\rangle \cap \mathcal{B}(r)$ has dimension more than one, because then the spread elements $\mathcal{B}(r)$ and $\mathcal{B}(s)$ would intersect, so $L_{r}$ is a line.

Now $\left\langle L_{r}, \pi\right\rangle$ meets $\mathcal{B}(s)$ in a line $L_{s}$ since the 3 -dimensional space $\left\langle L_{r}, \pi\right\rangle$ is contained in the $h+1$-space $\langle\pi, \mathcal{B}(s)\rangle$ and $\mathcal{B}(s)$ is $h-1$-dimensional. Using 
the same reasoning as above, we get that $\left\langle L_{r}, \pi\right\rangle \cap \mathcal{B}(s)$ cannot have dimension larger than one. Hence, $\left\langle\pi, L_{r}\right\rangle$ intersects both $\mathcal{B}(s)$ and $\mathcal{B}(r)$ in a line. Suppose that there is a 3-dimensional space $\mu^{\prime}$, intersecting $\mathcal{B}(r)$ in the line $L_{r}^{\prime}$ and $\mathcal{B}(s)$ in the line $L_{s}^{\prime}$, then $L_{r}^{\prime}$ is the intersection $L_{r}$ of $\langle\mathcal{B}(s), \pi\rangle$ with $\mathcal{B}(r)$ and $L_{s}^{\prime}$ is the intersection $L_{s}$ of $\langle\mathcal{B}(r), \pi\rangle$ with $\mathcal{B}(s)$. Hence, $\mu$ is uniquely determined.

(2) Suppose that there is a line $L$ through $r, L \notin \pi$, such that $\mathcal{B}(s) \in \mathcal{B}(L)$ and $\mathcal{B}(L)$ is contained in $\pi$. An element $\mathcal{B}(x) \in \mathcal{B}(L)$ intersects $\langle\pi, L\rangle$ in the line $L_{x}=\langle L \cap \mathcal{B}(x), \pi \cap \mathcal{B}(x)\rangle$. The $q$ lines $L_{x}, x \in L \backslash\{r\}$ belong to a 1-regulus with transversal line $L$, so $\mathcal{B}(r) \in \mathcal{B}(L)$ intersects $\langle\pi, L\rangle$ in a line too. (see Lemma 10)

Corollary 15. Through two points of a scattered linear set $\mathcal{B}(\pi)$ of rank 3 in $\mathrm{PG}\left(1, q^{h}\right), q>2$, there are at most two sublines contained in $\mathcal{B}(\pi)$. If $h=3$, through two points of $\mathcal{B}(\pi)$, there are exactly two sublines contained in $\mathcal{B}(\pi)$.

Proof. Let $\mathcal{B}(\pi)$ be a scattered linear set of rank 3 and let $r, s \in \pi$. The subline $\mathcal{B}(r s)$ is contained in $\mathcal{B}(\pi)$. By Lemma $14(1)$, there is a unique space 3-dimensional space $\left\langle L_{r}, L_{s}\right\rangle, L_{r} \in \mathcal{B}(r), L_{s} \in \mathcal{B}(s)$ through $\pi$. If there are exactly two elements of $\mathcal{D}$ that intersect the space $\left\langle L_{r}, L_{s}\right\rangle$ in a line, there are no irregular sublines, if there are $q+1$ elements of $\mathcal{D}$ that intersect the space $\left\langle L_{r}, L_{s}\right\rangle$ there is an irregular subline through $\mathcal{B}(r)$ and $\mathcal{B}(s)$. Lemma $14(2)$ shows that if there is an irregular subline, this irregular subline is unique.

Lemma 10 shows that if $h=3$, there are always $q+1$ elements of $\mathcal{D}$ intersecting $\left\langle L_{r}, L_{s}\right\rangle$ in a line.

Remark 16. Through two points of a scattered linear set $\mathcal{B}(\pi)$ of rank 3 in $\mathrm{PG}\left(1,2^{h}\right)$, there are exactly 5 sublines contained in $S$. Let $P, R$ be two points of $\mathcal{B}(\pi)$. Through every of the 5 points $Q_{i}$, different from $P$ and $R$, contained in $\mathcal{B}(\pi)$, there is exactly one subline containing $P, R$ and $Q_{i}$. Since $q=2$, this subline only contains the points $P, R, Q_{i}$, hence, is completely contained in $S$.

\subsection{Irregular sublines as the projection of a subconic in $\mathrm{PG}\left(2, q^{3}\right)$}

Using Theorem 1, we see that a linear set $S$ of rank 3 in $\operatorname{PG}\left(2, q^{3}\right)$ is the projection of a subplane $\mathrm{PG}(2, q)$ from a point in $\mathrm{PG}\left(2, q^{3}\right) \backslash \mathrm{PG}(2, q)$. The projection of a line of $\operatorname{PG}(2, q)$ is a subline of $S$. The irregular sublines are sublines that are not the projection of a line of $\operatorname{PG}(2, q)$. In this section, we show that an irregular subline is the projection of a conic, and we investigate when the projection of a conic is a subline.

Theorem 17. [6, Chapter 6] The points $(0,0,1),\left(0,1, x_{1}\right),\left(0,1, x_{2}\right),\left(0,1, x_{3}\right)$ of $\mathrm{PG}\left(2, q^{t}\right), q=p^{h}$ are contained in a subline over $\mathbb{F}_{q}$ iff $\frac{x_{2}-x_{1}}{x_{3}-x_{1}} \in \mathbb{F}_{q}$.

Lemma 18. The quotient space $C / P$ of an irreducible conic in $\mathrm{PG}(2, q)$ over a point $P$, where $P$ lies on $C^{*}$, and not on an extended line of $\mathrm{PG}(2, q)$, where $C^{*}$ denotes the extension of $C$ to $\mathrm{PG}\left(2, q^{3}\right)$ in $\mathrm{PG}\left(2, q^{3}\right) \backslash \mathrm{PG}(2, q)$ is a subline.

Proof. Let $C$ be an irreducible conic in $\operatorname{PG}(2, q)$. There is an element $\phi$ of $\operatorname{P\Gamma L}(3, q)$ that maps $C$ onto the conic $C^{\prime}:\left(1, a, a^{2}\right) \cup(0,0,1)$, where $a \in \mathbb{F}_{q}$. We will project $C^{\prime}$ on the line $X_{0}=0$ from a point $P=\left(1, \alpha, \alpha^{2}\right)$ on $C^{*}$, where $\alpha \in \mathbb{F}_{q^{3}} \backslash \mathbb{F}_{q}$. 
The projection of $C^{\prime}$ from $P$ on the line $X_{0}=0$ consists of the points $\left(0, \alpha-x, \alpha^{2}-x^{2}\right) \cup(0,0,1)$ for all $x \in \mathbb{F}_{q}$, which equals $(0,1, \alpha+x) \cup(0,0,1)$, since $\alpha \neq x$. Result 17 shows that the 4 points $(0,0,1),\left(0,1, \alpha+x_{1}\right),\left(0,1, \alpha+x_{2}\right)$, and $\left(0,1, \alpha+x_{3}\right)$ are on a subline iff

$$
\frac{\alpha+x_{2}-\alpha-x_{1}}{\alpha+x_{3}-\alpha-x_{1}} \in \mathbb{F}_{q}
$$

Since this equality holds for all $x_{i} \in \mathbb{F}_{q}$, the lemma follows.

Corollary 19. (i) The quotient space $C / P$ of an irreducible conic $C$ in $\mathrm{PG}(2, q)$ over a point $P$, not on an extended line of $\mathrm{PG}\left(2, q^{3}\right)$ in $\mathrm{PG}\left(2, q^{3}\right) \backslash \mathrm{PG}(2, q)$, is a subline if and only if $P$ lies on $C^{*}$, where $C^{*}$ denotes the extension of $C$ to $\operatorname{PG}\left(2, q^{3}\right)$.

(ii) The quotient space $C / P$ of an irreducible conic $C$ in $\mathrm{PG}(2, q)$ over a point $P$ in $\mathrm{PG}\left(2, q^{3}\right) \backslash \mathrm{PG}(2, q)$ on an extended line of $\mathrm{PG}(2, q)$, is not a subline.

Proof. (i) Corollary 15, with $h=3$, shows that there is exactly one irregular subline through 2 points $Q$ and $R$ of a scattered linear set $S$ of rank 3 . It is clear that the subline through the pre-images $Q^{\prime}$ and $R^{\prime}$ in $\operatorname{PG}(2, q)$ of $Q$ and $R$ by the projection from $P$, is projected onto a subline $L$ through $Q$ and $R$, contained in $S$.

Since Lemma 18 shows that the unique conic through the point $P, P^{q}$ and $P^{q^{2}}$ and the two points $Q$ and $R$ is projected onto a subline, different from $L$, the statement follows.

(ii) It is shown in Corollary 13 that there is no irregular subline contained in a club. Hence, the projection of a conic $C$ from a point on an extended line cannot be a subline.

\subsection{Sublines contained in a linear set}

In the following theorem, we show that every subline is an irregular subline of some linear set.

Theorem 20. For every $k \leq h+1$, and for every subline $\mathcal{B}(L)$ in $\mathrm{PG}\left(1, q^{h}\right)$, there is a linear set $\mathcal{B}(\pi)$ of rank $k$ such that $\mathcal{B}(L) \subset \mathcal{B}(\pi), L \not \subset \pi$, and such that $\mathcal{B}(L) \not \subset \mathcal{B}\left(\pi^{\prime}\right)$ for every proper subspace $\pi^{\prime}$ of $\pi$.

Proof. Let $\mathcal{B}(L)=\left\{\sigma_{1}, \ldots, \sigma_{q+1}\right\}$ be a subline of $\mathrm{PG}\left(1, q^{h}\right)$. Let $t_{P}$ denote the transversal line through a point $P$ to $\mathcal{B}(L)$. Let $P_{1}$ be a point of $\sigma_{1}$, let $P_{2}$ be a point in $\sigma_{2} \backslash t_{P_{1}}$, let $P_{3}$ be a point in $\sigma_{3} \backslash\left\langle t_{P_{1}}, t_{P_{2}}\right\rangle$, let $P_{i}, i \leq k-1$ be a point in $\sigma_{i} \backslash\left\langle t_{P_{1}}, \ldots, t_{P_{i-1}}\right\rangle$. Let $P_{k}$ be a point in $\sigma_{k} \cap\left\langle t_{P_{1}}, \ldots, t_{P_{k-1}}\right\rangle$, not in $\left\langle P_{1}, \ldots, P_{k-1}\right\rangle$.

The $k$-2-dimensional space $\left\langle t_{P_{1}} \cap \sigma_{i}, \ldots, t_{P_{k-1}} \cap \sigma_{i}\right\rangle \subset \sigma_{i}$ and the $k-1$ space $\pi=\left\langle P_{1}, \ldots, P_{k}\right\rangle$ are both contained in the $2 k-3$-dimensional space $\left\langle\left\langle t_{P_{1}} \cap \sigma_{1}, \ldots, t_{P_{k-1}} \cap \sigma_{1}\right\rangle,\left\langle t_{P_{1}} \cap \sigma_{k}, \ldots, t_{P_{k-1}} \cap \sigma_{k}\right\rangle\right\rangle$. Hence, $\sigma_{i}$ meets $\pi$ for all $i$.

Since the points $P_{1}, \ldots, P_{k}$ span the space $\pi$ and are the intersection points of $\sigma_{k}$ with $\pi, \mathcal{B}(L)$ cannot be contained in $\mathcal{B}\left(\pi^{\prime}\right)$ with $\pi^{\prime}$ a proper subspace of $\pi$. 


\section{$5 \quad$ The intersection of two linear sets of rank 3}

In [5], the authors show that two different linear sets of rank 3 in $\mathrm{PG}\left(1, q^{3}\right)$ share at most $2 q+2$ points, where $q=p^{h}, p \geq 7$, using coordinates and the fact that linear sets of the same size in $\mathrm{PG}\left(1, q^{3}\right)$ are projectively equivalent. Now, we prove this in a geometrical way and extend this result, at least for odd $q$, to $\mathrm{PG}\left(1, q^{h}\right)$. Moreover, we show that the bound is sharp.

Lemma 21. Let $\pi$ and $\pi^{\prime}$ be two planes (in $\mathrm{PG}(r h-1, q)$ ) with $\pi \cap \pi^{\prime}=L, L$ a line. Let $\mathcal{D}$ be a Desarguesian $(h-1)$-spread in $\mathrm{PG}($ rh $-1, q)$. If $\mathcal{B}(\pi) \neq \mathcal{B}\left(\pi^{\prime}\right)$, then $\left|\mathcal{B}(\pi) \cap \mathcal{B}\left(\pi^{\prime}\right)\right|=1,2, q+1, q+2, q+3,2 q+1,2 q+2$.

Proof. By Lemma 10, the number of elements of $\mathcal{D}$ intersecting the 3-dimensional space $\left\langle\pi, \pi^{\prime}\right\rangle$ in a line is 1,2 or $q+1$ since $\mathcal{B}(\pi) \neq \mathcal{B}\left(\pi^{\prime}\right)$. If $\left|\mathcal{B}\left(\pi \cap \pi^{\prime}\right)\right|=1$, this implies that $\mathcal{B}(\pi)$ and $\mathcal{B}\left(\pi^{\prime}\right)$ have 1,2 or $q+1$ elements in common. If $\left|\mathcal{B}\left(\pi \cap \pi^{\prime}\right)\right|=q+1$, and $\pi$ or $\pi^{\prime}$ is a club, $\left|\mathcal{B}(\pi) \cap \mathcal{B}\left(\pi^{\prime}\right)\right|=q+1, q+2$ or $2 q+1$. If $\left|\mathcal{B}\left(\pi \cap \pi^{\prime}\right)\right|=q+1$ and $\pi$ and $\pi^{\prime}$ is not a club, this implies that $\mathcal{B}(\pi)$ and $\mathcal{B}\left(\pi^{\prime}\right)$ have $q+1, q+2, q+3,2 q+1$ or $2 q+2$ elements in common

Theorem 22. Two $\mathbb{F}_{q}$-linear sets of rank 3 in $\mathrm{PG}\left(1, q^{h}\right), q>3$, intersect in at most $2 q+2$ points if $q$ is odd, and in at most $2 q+3$ points if $q$ is even.

Proof. Let $\mathcal{D}$ be a Desarguesian $(h-1)$-spread in $\mathrm{PG}(r h-1, q), q>3$. Let $\pi$ and $\pi^{\prime}$ be two planes (in $\mathrm{PG}(r h-1, q)$ ) with $\pi \cap \pi^{\prime} \neq \emptyset$ and suppose that $\left|\mathcal{B}(\pi) \cap \mathcal{B}\left(\pi^{\prime}\right)\right| \geq 2 q+3$. Let $X=\left\{P\right.$ a point $\left.\in \pi \mid \mathcal{B}(P) \subset \mathcal{B}\left(\pi^{\prime}\right)\right\}$ and $X^{\prime}=$ $\left\{P\right.$ a point $\left.\in \pi^{\prime} \mid \mathcal{B}(P) \subset \mathcal{B}(\pi)\right\}$. From Theorem 8, we get that if a line contains 4 points of $X$ (resp. $X^{\prime}$ ), then this line is contained in $X$ (resp. $X^{\prime}$ ). If $\pi \cap \pi^{\prime}$ is a line, the theorem follows from Lemma 21 , hence, suppose that $\pi$ intersects $\pi^{\prime}$ in the point $P$.

Suppose first that there are no lines in $X$ or $X^{\prime}$, say in $X$. Then every line in $X$ contains at most 3 points of $X$. In that case, $|X|=2 q+3$, and every line intersects $X$ in 0 or 3 points and $q=3^{h}$. But a maximal arc in a plane of odd order does not exist (see [1]), a contradiction. Hence, from now on, we assume that there is a line $L_{X}$ in $X$ and a line $L_{X^{\prime}}$ in $X^{\prime}$.

Case 1: $\left|\mathcal{B}\left(L_{X}\right)\right|=1$. The elements of $\mathcal{B}\left(L_{X}^{\prime}\right)$ meet the plane $\pi$ in points of a conic $C$. Since $|X| \geq 2 q+3$, there is a point $P_{1}$ contained in $X \backslash\left\{C \cup L_{X}^{\prime}\right\}$ lying on a secant line $M$ to $C$ which meets $L_{X}$ in a point not on $C$, hence containing 4 points of $X$, which implies that $M \subset X$. Now if $q>3$, every point of $\pi$ lies on a secant line to $C$, intersecting $L_{X}$ and $M$ in a point. This shows that $\pi=X$.

Case 2: $\left|\mathcal{B}\left(L_{X}\right)\right|=q+1$. Hence, the elements of $\mathcal{B}\left(L_{X}\right)$ meet $\pi^{\prime}$ in the points of a conic $C$.

Let $P_{1}$ be a point of $C$, and let $Q$ be the point on the line $L_{X}$ such that $\mathcal{B}(Q)=\mathcal{B}\left(P_{1}\right)$. There is a plane $\pi^{\prime \prime}$, through $P_{1}$, such that $\mathcal{B}(\pi)=\mathcal{B}\left(\pi^{\prime \prime}\right)$, moreover, the plane $\pi$ " contains a line $L$ through $P_{1}$ with $\mathcal{B}(L)=\mathcal{B}\left(L_{X}\right)$. The elements of $\mathcal{B}\left(L_{X^{\prime}}\right)$ meet $\pi$ " in the points of a conic $C^{\prime}$. Since $|X| \geq 2 q+3$, $X$ contains a point $Q$, not on $L \cup C^{\prime}$. If there is a secant line $M$ through $Q$ to $C^{\prime}$, not through the possible intersection of $L$ with $C$, containing 4 points of $X$, then $M$ is contained in $X$. It is easy to see that if $q>3$, every point $R$ of $\pi$, different from the nucleus $n$ of $C^{\prime}$ if $q$ is even, lies on a secant line through $C^{\prime}$, meeting $M$ and $L$. But then $R$ lies on a line with four points of $X$, and we 
conclude that $X=\pi$. If $q$ is odd, the secant line $M$ always exists. If $q$ is even, it is possible that $Q$ is the nucleus of $C^{\prime}$. In the latter case, if $|X| \geq 2 q+4$, there is a point $Q^{\prime} \in X$, lying on a secant line $M$ to $C^{\prime}$ and we can repeat the previous argument with $Q=Q^{\prime}$ to show that $X=\pi$. Now the statement follows.

Remark 23. For general $q$, there are two linear sets of rank 3, intersecting in $2 q+2$ points. Let $\pi$ be a 3-dimensional space, such that there are $q+1$ elements of $\mathcal{D}$, say $\sigma_{i}$ intersecting $\pi$ in a line $L_{i}$. Let $M$ be a line skew to all lines $L_{i}$. Let $\pi$ and $\pi^{\prime}$ be two different planes through $M$. The sets $\mathcal{B}(\pi)$ and $\mathcal{B}\left(\pi^{\prime}\right)$ have exactly $2 q+2$ points in common.

\section{References}

[1] S. Ball, A. Blokhuis, F. Mazzocca. Maximal arcs in Desarguesian planes of odd order do not exist. Combinatorica 17 (1997), 31-41.

[2] A. Blokhuis, M. Lavrauw. Scattered spaces with respect to a spread in PG $(n, q)$. Geom. Dedicata 81, no. 1-3, (2000), 231-243.

[3] G. Donati, N. Durante. On the intersection of two subgeometries of PG $(n, q)$. Des. Codes Cryptogr. 46 (2008), 261-267.

[4] Sz. Fancsali, P. Sziklai. About maximal partial 2-spreads in PG(3m-1, $)$. Innov. Incidence Geom. 4 (2006), 89-102.

[5] S. Ferret, L. Storme. Results on maximal partial spreads in $\mathrm{PG}\left(3, p^{3}\right)$ and on related minihypers. Des. Codes Cryptogr. 29 (2003), 105-122.

[6] J.W.P. Hirschfeld. Projective geometries over finite fields. Second edition, Oxford University Press, New York, 1998. xiv+555 pp.

[7] M. Lavrauw, O. Polverino. Finite Semifields. Chapter to appear in Current research topics in Galois geometries,

[8] M. Lavrauw, L. Storme, G. Van de Voorde. A proof for the linearity conjecture for $k$-blocking sets in $\operatorname{PG}\left(n, p^{3}\right), p$ prime. Submitted.

[9] G. Lunardon. Normal spreads. Geom. Dedicata 75 (1999), 245-261.

[10] G. Lunardon, O. Polverino. Translation ovoids of orthogonal polar spaces. Forum Math. 16 (2004), 663-669.

[11] O. Polverino. Linear sets in finite projective spaces. Discrete Math (2009). In press.

[12] P. Sziklai. On small blocking sets and their linearity. J. Combin. Theory, Ser. A, 115 (2008), no. 7, 1167-1182. 